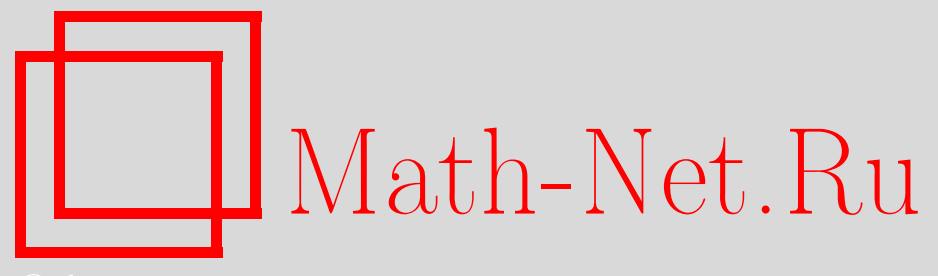

В. Д. Матвеенко, Структура оптимальных траекторий дискретной детерминированной схемы с дисконтированием, Дискрет. матем., 1998, том 10, выпуск 3, 100-114

DOI: https://doi.org/10.4213/dm437

Использование Общероссийского математического портала Math-Net.Ru подразумевает, что вы прочитали и согласны с пользовательским соглашением http://www . mathnet.ru/rus/agreement

Параметры загрузки:

IP: 54.210 .77 .194

26 апреля 2023 г., 12:59:36

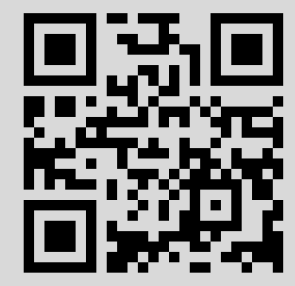




\section{Структура оптимальных траекторий дискретной детерминированной схемы с дисконтированием}

(C) 1998 г. В. Д. Матвеенко

Исследуется семейство задач нахождения

$$
\max _{i_{1}, \ldots, i_{T-1}} \sum_{k=0}^{T-1} \beta^{k} u\left(i_{k}, i_{k+1}\right)
$$

при $i_{0}=j_{0}, i_{T}=j_{T}$, где $\beta$ - дисконтирующий множитель $(\beta>0, \beta \neq 1) ; i_{k}$, $k=0,1, \ldots, T$, - элементы заданного конечного множества; $u$ - функция, принимающая значения в пространстве $\mathbf{R} \cup\{-\infty\}$. Число шагов $T$ и краевые состояния $j_{0}, j_{T}$ рассматриваются как параметры. Дано описание структуры оптимальных траекторий при достаточно большом числе шагов $T$. Доказана теорема о представлении функции-значения. Указано достаточное условие, при выполнении которого данның контур не входит в состав никакой оптимальной траектории, независимо от величины $\beta$.

\section{1. Введение}

Статья посвящена изучению структуры оптимальных траекторий детерминированной управляемой системы с дискретным множеством состояний с дисконтированием. Подобные задачи без дисконтирования рассматривались, например, в [1-6]. В частности, было показано (см. [5]), что при определенных условиях, близких по смыслу к неразложимости и примитивности матрицы, всякая достаточно длинная $T$-шаговая оптимальная траектория с фиксированными концами состоит из трех последовательных участков : первый участок зависит лишь от начального состояния (не зависит от финального состояния и от длины траектории) и совпадает с соответствующим участком бесконечной оптимальной траектории ; на втором участке траектория проходит по асимптотически устойчивому магистральному множеству ; третий участок зависит лишь от финального состояния и совпадает с соответствующим участком оптимальной обратной траектории. Аналогичная структура оптимальных траекторий была обнаружена и для некоторых других управляемых систем (см. $[7,8])$. Однако следующий естественный шаг, рассмотрение дискретной системы при наличии дисконтирующего множителя, сделан не был. Ликвидировать отчасти этот пробел и составляет цель статьи. 
Пусть задано конечное множество $M$, состоящее из $n$ элементов. В дальнейшем будем называть его множеством вершин. Задана также функция

$$
u: M \times M \rightarrow \mathbf{R} \cup\{-\infty\} .
$$

Значение $u(i, j)$ будем называть полезностью или длиной дуги $(i, j)$. Будем считать, что граф $G$, составленный из дуг $(i, j)$, для которых $u(i, j)>-\infty$, вполне связен. Задано также число $\beta>0$, называемое дисконтирующим множителем или дисконтом. Наконец, фиксированы две вершины $j_{0}, j_{T}$ и натуральное число $T$. Требуется максимизировать функцию

$$
\sum_{k=0}^{T-1} \beta^{k} u\left(i_{k}, i_{k+1}\right)
$$

на множестве всевозможных наборов вершин ( $T$-шаговых траекторий)

$$
\left\{i_{0}=j_{0}, i_{1}, i_{2}, \ldots, i_{T}=j_{T}\right\}
$$

Указанную задачу будем обозначать символом $S_{\beta}\left(j_{0}, j_{T}, T\right)$. При изучении структуры решений будем рассматривать семейство задач $S_{\beta}\left(j_{0}, j_{T}, T\right)$ при различных значениях параметров $j_{0}, j_{T}, T$.

При $0<\beta<1$ с задачами $S_{\beta}\left(j_{0}, j_{T}, T\right)$ тесно связано другое семейство задач, в которых фиксирована начальная вершина и требуется найти бесконечную последовательность вершин (бесконечную траекторию)

$$
\left\{i_{0}=j_{0}, i_{1}, i_{2}, \ldots\right\}
$$

максимизирующую функцию

$$
\sum_{k=0}^{\infty} \beta^{k} u\left(i_{k}, i_{k+1}\right)
$$

Такие задачи будут обозначаться как $S_{\beta}^{\infty}\left(j_{0}\right)$.

Семейство задач $S_{\beta}\left(j_{0}, j_{T}, T\right)$ имеет непосредственные приложения в исследовании операций (например, выбор последовательности работ). Кроме того, эти задачи состоят в естественном родстве с аналогичными задачами с непрерывным пространством состояний, которые имеют многочисленные приложения в математической экономике. Среди этих приложений широко известная модель Рамсея в ее вариантах с дискретным и с непрерывным временем (см., например, $[9,10])$ и ее $n$-мерные аналоги (см. $[11,12])$. Задачи, родственные $S_{\beta}^{\infty}\left(j_{0}\right)$ изучались также в контексте управляемых стохастических процессов (см., например, [13]) и динамических игр (например, [14]). Хотя в настоящей работе рассматривается дискретное множество состояний, ее результаты могут оказаться полезными и при изучении систем с непрерывным пространством состоянић. Во-первых, все три класса моделей (с дискретным множеством состояний, с непрерывным множеством состояний и дискретным временем, с непрерывным множеством состояний и непрерывным временем) связаны идейно и часто дают параллельные результаты. Во-вторых, модели одного класса могут применяться как математический аппарат для исследования моделей другого класса. Например, система с непрерывным пространством состояний и дискретным временем может быть аппроксимирована системой с дискретным пространством состояний (см., например, [15]). 
Полученные в этой статье результаты напоминают результаты для случая отсутствия дисконтирования $(\beta=1)$. В то же время имеются существенные отличия.

При $\beta=1$ задача $S_{1}^{\infty}\left(j_{0}\right)$, вообще говоря, не имеет решения. Вводят модифицированную функцию полезности

$$
\bar{v}(i, j)=v(i, j)-\lambda, \quad i, j \in M \text {. }
$$

Здесь $\lambda$ - максимальная средняя длина дуги, найденная по всевозможным контурам графа $G$ (максимальная характеристика, в терминологии $[1,5]$ ), она играет роль, аналогичную собственному числу матрицы (см., [5]). С этой новой функцией полезности естественным образом определены задачи $\bar{S}_{1}\left(j_{0}, j_{T}, T\right)$ и $\bar{S}_{1}^{\infty}\left(j_{0}\right)$. Оказывается (см., например, [5]), что решения задач $S_{1}\left(j_{0}, j_{T}, T\right)$ и $\bar{S}_{1}\left(j_{0}, j_{T}, T\right)$ совпадают, а задача $\bar{S}_{1}^{\infty}\left(j_{0}\right)$ имеет решение. Пусть $V_{1}(i, j, T)$ и $V_{1}(i)-$ функции-значения, соответственно, задач $S_{1}(i, j, T)$ и $\bar{S}_{1}^{\infty}(i)$. Как показано в [5], при определенных условиях существует также функция $W_{1}(i)$ такая, что

$$
V_{1}\left(j_{0}, j_{T}, T\right)=\lambda T+V_{1}\left(j_{0}\right)+W_{1}\left(j_{T}\right), \quad j_{0}, j_{T} \in M
$$

при достаточно больших $T$.

В настоящей статье при $\beta<1$ исследование также основано на сравнении средних (теперь средних взвешенных) длин дуг контуров. Принципиальное отличие при $\beta<1$ состоит в том, что средняя длина дуги зависит от того, относительно какой вершины контура она определена. При этом отсутствует единая максимальная характеристика $\lambda$. С другой стороны, при $\beta<1$ задачи $S_{\beta}^{\infty}(i), i \in M$, имеют решение, таким образом, по ним непосредственно определена функция-значение $V_{\beta}(i)$.

В параграфе 2 при $\beta<1$ исследуются оптимальные контуры и структура траекторий-решений задачи $S_{\beta}^{\infty}\left(j_{0}\right)$. В параграфе 3 изучается структура траекторийрешений задачи $S_{\beta}\left(j_{0}, j_{T}, T\right)$. В частности, мы показываем, что при определенных условиях, существует такая функция $W_{\beta}(i)$, что, при достаточно больших $T$,

$$
V_{\beta}\left(j_{0}, j_{T}, T\right)=V_{\beta}\left(j_{0}\right)+\beta^{T} W_{\beta}\left(j_{T}\right), \quad j_{0}, j_{T} \in M
$$

В параграфе 4 приводится численный пример. В параграфе 5 показано, каким образом случай $\beta>1$ может быть сведен к случаю $\beta<1$.

\section{2. Бесконечные оптимальные траектории}

Пусть $V_{\beta}(i)$ - значения задач $S_{\beta}^{\infty}(i), i \in M ; V_{\beta}(i, j, T)$ - значения задач $S_{\beta}(i, j, T)$, $i, j \in M, T=1,2, \ldots$, при фиксированном $\beta \in(0,1)$. Из принципа оптимальности Беллмана следует, что эти функции удовлетворяют, соответственно, уравнениям Беллмана

$$
\begin{aligned}
V_{\beta}(i) & =\max _{j \in M}\left\{u(i, j)+\beta V_{\beta}(j)\right\}, \\
V_{\beta}(i, j, T) & =\max _{\gamma \in M}\left\{u(i, \gamma)+\beta V_{\beta}(\gamma, j, T-1)\right\}, \quad T>1 .
\end{aligned}
$$

Бесконечная траектория $\left\{i_{0}, i_{1}, \ldots\right\}$ называется пошагово оптимальной, если

$$
V_{\beta}\left(i_{t}\right)=u\left(i_{t}, i_{t+1}\right)+\beta V_{\beta}\left(i_{t+1}\right), \quad t=0,1, \ldots,
$$


T. e.

$$
i_{t+1} \in \operatorname{Arg} \max _{j \in M}\left\{u\left(i_{t}, j\right)+\beta V_{\beta}(j)\right\} .
$$

Известно следующее свойство (см. [16]), справедливое как для дискретного, так и для непрерывного множества состояний.

Теорема 1. Бесконечная траектория $\left\{i_{0}, i_{1}, \ldots\right\}$ оптимальна (является решением задачи $\left.S_{\beta}^{\infty}\left(i_{0}\right)\right)$ тогда и толъко тогда, когда она пошагово оптималъна.

Замечание 1. При малых положительных дисконтах $\beta$ оптимальные траектории можно охарактеризовать непосредственно. Назовем лексикографически максимальной (л.м.) такую траекторию $\left\{j_{0}, j_{1}, \ldots\right\}$, что для любой траектории $\left\{i_{0}=j_{0}, i_{1}, \ldots\right\}$ выполняется следуюшее: если $u\left(i_{t}, i_{t+1}\right)>u\left(j_{t}, j_{t+1}\right)$ для некоторого индекса $t$, то найдется индекс $k<t$, для которого $u\left(i_{k}, i_{k+1}\right)<u\left(j_{k}, j_{k+1}\right)$. Нетрудно показать, что существует такое значение $\bar{\beta}$, что при всех дисконтах $\beta \in(0, \bar{\beta})$ оптимальными являются л. м. траектории и только они. Имеется простой алгоритм построения л. м. траекторий, на котором мы здесь не останавливаемся. Отметим лишь, что всякая л. м. траектория $\left\{j_{t}\right\}$ является пошагово оптимальной при $\beta=0$, т.е.

$$
j_{t+1} \in \operatorname{Arg} \max _{i \in M} u\left(j_{t}, i\right), \quad t=0,1, \ldots
$$

Обратное верно, если множества $\operatorname{Arg} \max _{i \in M} u(j, i), j \in M$, одноэлементные.

Замечание 2. Можно показать, что существует такое число $\tilde{\beta}<1$, что при всех дисконтах $\beta \in(\tilde{\beta}, 1)$ всякая траектория, являющаяся решением задачи $S_{\beta}^{\infty}\left(j_{0}\right)$, является одновременно и решением задачи $\bar{S}_{1}^{\infty}\left(j_{0}\right)$ (см. параграф 1$)$. Обратное, вообще говоря, неверно.

По поводу влияния величины дисконта на асимптотику оптимальных траекторий в моделях с непрерывным пространством состояний см. $[17,18]$.

В силу конечности множества $M$, очевидно, что всякая достаточно длинная (и, тем более, бесконечная) траектория содержит контуры. Нам понадобятся некоторые свойства суммарных дисконтированных полезностей, рассчитанных по контуру. Пусть $\sigma=\left\{i_{1}, i_{2}, \ldots, i_{|\sigma|}, i_{1}\right\}-$ контур, состоящий из $|\sigma|$ дуг. Фиксирован дисконт $0<\beta<1$. Дисконтированной полезностью контура $\sigma$ относительно вершины $i_{s}$ назовем число

$$
\chi_{\sigma, i_{s}}=\sum_{h=s}^{s+|\sigma|-1} \beta^{h-s} u\left(i_{h}, i_{h+1}\right),
$$

где $i_{|\sigma|+k}=i_{k}$. Заметим, что

$$
\chi_{\sigma, i_{s}}=u\left(i_{s}, i_{s+1}\right)\left(1-\beta^{|\sigma|}\right)+\beta \chi_{\sigma, i_{s+1}}
$$

при $\beta=1$ эта формула дала бы $\chi_{\sigma, i_{s}}=\chi_{\sigma, i_{s+1}}$. Средней длиной дуги контура $\sigma$ относительно вершины $i_{s}$ естественно назвать число $c_{\sigma, i_{s}}$ такое, что

$$
\left(1+\beta+\beta^{2}+\ldots+\beta^{|\sigma|-1}\right) c_{\sigma, i_{s}}=\chi_{\sigma, i_{s}} .
$$

Отсюда

$$
c_{\sigma, i_{s}}=\chi_{\sigma, i_{s}} \frac{1-\beta}{1-\beta^{|\sigma|}}
$$


Легко найти и дисконтированную полезность, получаемую при бесконечных обходах контура $\sigma$, начиная с вершины $i_{s}$ :

$$
v_{\sigma, i_{s}}=\chi_{\sigma, i_{s}}\left(1+\beta^{|\sigma|}+\beta^{2|\sigma|}+\ldots\right)=\frac{\chi_{\sigma, i_{s}}}{1-\beta^{|\sigma|}} .
$$

В силу (4)

$$
v_{\sigma, i_{s}}=\frac{c_{\sigma, i_{s}}}{1-\beta}
$$

Смысл этого равенства в том, что бесконечные обходы контура $\sigma$ можно было бы заменить бесконечными обходами петли с полезностью $c_{\sigma, i}$ (если бы такая петля существовала).

Контур $\sigma$ называется оптимальным относительно вершины $j_{0} \in M$, если существует бесконечная оптимальная траектория с началом $j_{0}$ (решение задачи $S_{\beta}^{\infty}\left(j_{0}\right)$ ), включающая хотя бы один обход контура $\sigma$.

Лемма 1. Если $i$ - вершина контура $\sigma u$

$$
V_{\beta}(i)=\frac{C_{\sigma, i}}{1-\beta}
$$

то контур б оптимален относителъно $i$.

Доказательство. Правая часть равенства представляет собой полезность траектории $\tau$ с началом $i$, состоящей из бесконечного обхода контура $\sigma$. Равенство означает, что траектория $\tau$ оптимальна, т. е. контур $\sigma$ оптимален относительно $i$.

Лемма доказана.

Обратное лемме 1 утверждение составляет часть следующего предложения.

Предложение 1. Следующие утверждения эквивалентны:

(1) хонтур $\sigma$ оптимален относителъно нехоторой вериины $j_{0} \in M$;

(2) контур б оптимален относителъно каждой своей вершинъ $j$;

(3) существует оптимальная траектория $\tau^{*}$ с началом $j_{0}$, заканчивающаяся бесконечным обходом контура $\sigma$;

(4) $V(j)=C_{\sigma, j} /(1-\beta)$ для любой вершины $j$ контура $\sigma$;

(5) $V(j)=u(j, \tilde{\jmath})+\beta V(\tilde{\jmath})$ для любой дуги $(j, \tilde{\jmath})$ контура $\sigma$.

Доказательство. Переходы $2 \rightarrow 1$ и $3 \rightarrow 1$ тривиальны; из 4 следует 2 согласно лемме 1.

Докажем, что $1 \rightarrow 3$. В силу оптимальности $\sigma$, существует оптимальная траектория, состоящая из трех участков :

(1) перехода из вершины $j_{0}$ в вершину $i \in \sigma$; пусть эта конечная траектория имеет полезность $s\left(j_{0}, i\right)$ и состоит из $\left|s\left(j_{0}, i\right)\right|$ дуг;

(2) одного обхода контура $\sigma$; 
(3) бесконечной оптимальной траектории с началом $i$.

Соответственно,

$$
V_{\beta}\left(j_{0}\right)=s\left(j_{0}, i\right)+\beta^{\left|s\left(j_{0}, i\right)\right|} \chi_{\sigma, i}+\beta^{\left|s\left(j_{0}, i\right)\right|} V_{\beta}(i) .
$$

С другой стороны, в силу принципа оптимальности,

$$
V_{\beta}\left(j_{0}\right)=s\left(j_{0}, i\right)+\beta^{\left|s\left(j_{0}, i\right)\right|} V_{\beta}(i) .
$$

Из (6), (7) вытекает, что

$$
V_{\beta}(i)=\chi_{\sigma, i}+\beta^{|\sigma|} V_{\beta}(i) .
$$

Следовательно, с учетом (4),

$$
V_{\beta}(i)=\frac{\chi_{\sigma, i}}{1-\beta^{|\sigma|}}=\frac{c_{\sigma, i}}{1-\beta} .
$$

Правая часть равенства (8) представляет собой полезность траектории $\bar{\tau}$ с началом $i$, состоящей из бесконечного обхода контура $\sigma$. Заменяя $V_{\beta}(i)$ в $(7)$, приходим к выводу, что траектория $\tau^{*}$, составленная из первого из трех указанных выше участков и $\bar{\tau}$, оптимальна.

Переход $1 \rightarrow 5$ следует из оптимальности траектории $\bar{\tau}$.

Докажем, что $5 \rightarrow 4$. Для контура $\sigma$, домножая равенства

$$
V_{\beta}\left(i_{t}\right)=u\left(i_{t}, i_{t+1}\right)+\beta V_{\beta}\left(i_{t+1}\right), \quad t=1,2, \ldots,|\sigma|,
$$

соответственно, на $\beta^{t}$ и суммируя, получим, с учетом (4), что

$$
V_{\beta}\left(i_{1}\right)=\frac{c_{\sigma, i_{1}}}{1-\beta} \text {. }
$$

Предложение доказано.

Аналогично доказывается, что набор чисел $V(i), i \in M$, единственный, удовлетворяющий равенствам (2).

Лемма 2. Пусть контур $\sigma$ оптимален относителъно вершины $j_{0} \in M u$ содержит вершину $i$. Тогда всякий контур $\sigma^{1}$, оптималъный относителъно $i$, является такюе оптимальным относительно $j_{0}$.

Доказательство. Как показывает предложение 1, существует оптимальная траектория $\tau^{*}$, начальный участок которой представляет собой путь из $j_{0}$ в $i$. Продолжим этот участок, дополнив его оптимальной траекторией с началом $i$, содержащей, по крайней мере, один обход контура $\sigma^{1}$. Новая траектория с началом $j_{0}$ оптимальна (она имеет ту же полезность, что и $\tau^{*}$ ). Следовательно, контур $\sigma^{1}$ оптимален относительно $j_{0}$.

Лемма доказана.

Лемма 3. Всякий контур $\sigma$, составленный из дуг контуров, оптимальных относительно вершины $j_{0} \in M$, сам является оптимальным относителъно $j_{0}$. 
Доказательство. Согласно предложению 1 для каждой дуги $(i, j)$ контура $\sigma$ справедливо равенство

$$
V(i)=u(i, j)+\beta V(j) .
$$

Из предложения 1 следует, что контур $\sigma$ оптимален относительно каждой своей вершины, и из леммы 2 вытекает доказываемое утверждение.

Лемма доказана.

Рассмотрим подграф $\Gamma$, состоящий из дуг всевозможных оптимальных (относительно своих собственных вершин) контуров. В силу леммы 3 он распадается на (максимальные) вполне связные компоненты $\Gamma_{1}, \ldots, \Gamma_{s}$, которые мы будем называть контурными компонентами. Если Г вполне связен, то он сам составляет контурную компоненту.

Для контура $\sigma$ обозначим через $M^{-}(\sigma)$ множество вершин, относительно которых контур $\sigma$ оптимален. Доказанные утверждения показывают, что

для каждой вершины $j \in M$ найдется по крайней мере один контур $\sigma$ такой, что $j \in M^{-}(\sigma)$;

если контур $\sigma$ таков, что $M^{-}(\sigma) \neq \varnothing$, то $i \in M^{-}(\sigma)$ для каждой вершины $i \in \sigma$; если $\sigma_{1}, \sigma_{2}-$ контуры, принадлежащие одной контурной компоненте $\Gamma_{k}$, то

$$
M^{-}\left(\sigma_{1}\right)=M^{-}\left(\sigma_{2}\right) \text {. }
$$

Это значит, что все контуры, принадлежащие контурной компоненте $\Gamma_{k}$, являются оптимальными относительно одного и того же набора начальных вершин, который будем обозначать $M^{-}\left(\Gamma_{k}\right)$. Если всего имеется $s$ контурных компонент $\Gamma_{1}, \ldots, \Gamma_{s}$, то

$$
M^{-}\left(\Gamma_{1}\right) \cup \ldots \cup M^{-}\left(\Gamma_{s}\right)=M
$$

Какова структура бесконечной оптимальной траектории $\tau$ с началом $j_{0}$ (решения задачи $\left.S_{\beta}^{\infty}\left(j_{0}\right)\right)$ ? Возможно несколько случаев.

Если контурная компонента $\Gamma_{k}$, для которой $j_{0} \in M^{-}\left(\Gamma_{k}\right)$, единственна, то траектория $\tau$ выходит на $\Gamma_{k}$ и далее может двигаться произвольным образом по дугам этой контурной компоненты. Движение будет заведомо регулярным (нехаотичным), лишь если $\Gamma_{k}$ представляет собой контур (в частности, петлю).

Если существует несколько контурных компонент $\Gamma_{k}$, для которых $j_{0} \in M^{-}\left(\Gamma_{k}\right)$, то для каждой из них можно указать продолжающуюся в этой компоненте бесконечную оптимальную траекторию с началом $j_{0}$.

Контурные компоненты могут следовать друг за другом. Пусть $\sigma_{1}, \sigma_{2}-$ два оптимальных (относительно своих вершин) контура. Если $i \in \sigma_{1}$ и $i \in M^{-}\left(\sigma_{2}\right)$, то будем говорить, что контур $\sigma_{2}$ следует за $\sigma_{1}$ и использовать обозначение $\sigma_{1} \rightarrow \sigma_{2}$. Нетрудно установить следующие свойства.

Если $\sigma_{1} \rightarrow \sigma_{2}$, то $M^{-}\left(\sigma_{1}\right) \subset M^{-}\left(\sigma_{2}\right)$.

Контуры $\sigma_{1}, \sigma_{2}$ принадлежат одной контурной компоненте тогда и только тогда, когда $\sigma_{1} \rightarrow \sigma_{2}, \sigma_{2} \rightarrow \sigma_{1}$.

Если $\Gamma_{1}, \Gamma_{2}$ - контурные компоненты, и $\sigma_{1} \in \Gamma_{1}, \sigma_{2} \in \Gamma_{2}, \sigma_{1} \rightarrow \sigma_{2}$, то $\hat{\sigma}_{1} \rightarrow \hat{\sigma}_{2}$ для любых контуров $\hat{\sigma}_{1} \in \Gamma_{1}, \hat{\sigma}_{2} \in \Gamma_{2}$. Будем говорить, что контурная компонента $\Gamma_{2}$ следует за $\Gamma_{1}$ и использовать обозначение $\Gamma_{1} \rightarrow \Gamma_{2}$.

Если $\Gamma_{1} \rightarrow \Gamma_{2}$, то $M^{--}\left(\Gamma_{1}\right) \subset M^{-}\left(\Gamma_{2}\right)$. В этом случае оптимальная траектория может сколь угодно долго находиться в контурной компоненте $\Gamma_{1}$, а затем перейти в $\Gamma_{2}$. 
В общем случае решение задачи $S_{\beta}^{\infty}\left(j_{0}\right)$ имеет нерегулярный характер. В конкретных задачах, однако, часто встречается простейший случай, когда граф Г представляет собой петлю (см. далее пример в параграфе 4).

В приложениях часто бывает важно выделить стационарные состояния (неподвижные точки) динамической системы. В связи с этим интересен вопрос о достаточных условиях, при которых никакие иные контуры, кроме петель, невозможны на оптимальных траекториях. В [19] для некоторых моделей с непрерывным пространством состояний предложено условие, сходное с используемым ниже (9).

Теорема 2. Пусть для контура

$$
\sigma=\left\{i_{1}, i_{2}, \ldots, i_{|\sigma|}, i_{|\sigma|+1}=i_{1}\right\}
$$

с числом вершин $|\sigma|>1$ выполняется неравенство

$$
\sum_{s=1}^{|\sigma|} u\left(i_{s}, i_{s}\right)>\sum_{s=1}^{|\sigma|} u\left(i_{s}, i_{s+1}\right)
$$

Тогда при любом фиксированном $\beta \in(0,1)$ найдется такая вершина $i_{s} \in \sigma$, что

$$
c_{\sigma, i_{s}}<u\left(i_{s}, i_{s}\right)
$$

Доказательство. Предположим противное : пусть для некоторого $\beta \in(0,1)$ выполняется неравенство $c_{\sigma, i_{s}} \geqslant u\left(i_{s}, i_{s}\right)$ при всех $i_{s} \in \sigma$, или, в силу (4),

$$
\frac{1-\beta}{1-\beta^{|\sigma|}} \chi_{\sigma, i_{s}} \geqslant u\left(i_{s}, i_{s}\right), \quad i_{s} \in \sigma
$$

Суммируя неравенства (11) по $s=1, \ldots,|\sigma|$ и используя определение числа $\chi_{\sigma, i_{s}}$, получаем неравенство

$$
\sum_{s=1}^{|\sigma|} u\left(i_{s}, i_{s+1}\right) \geqslant \sum_{s=1}^{|\sigma|} u\left(i_{s}, i_{s}\right)
$$

что противоречит (9). Теорема доказана.

Следствие 1. Если контур б удовлетворяет неравенству (9), то он не является оптималъным ни для какой задачи $S_{\beta}^{\infty}\left(j_{0}\right), 0<\beta<1$.

Доказательство легко проводится на основе утверждения 2 предложения 1.

Следствие 2. Если (9) выполняется для любого контура $\sigma$ с числом $\partial у г ~|\sigma|>1$, то для любого $\beta \in(0,1)$ любой оптималъный контур является петлей.

В определенном смысле обратным теореме 2 является следующее утверждение.

Предложение 2. Пусть при некотором $\beta \in(0,1)$ для некоторого контура $\sigma$ въполняются неравенства (10) при всех $s=1, \ldots,|\sigma|$. Тогда контур $\sigma$ удовлетворяет неравенству (9). 


\section{3. Конечные оптимальные траектории}

Переходим к задачам $S_{\beta}\left(j_{0}, j_{T}, T\right)$. В отличие от задач $S_{\beta}^{\infty}\left(j_{0}\right)$ одной только полной связности графа $G$ недостаточно для существования решения при любом $T$. Может просто не оказаться ни одного пути длины $T$ с началом $j_{0}$. Возможно также, что оптимальные $T$-шаговые траектории ведут себя по-разному, в зависимости от кратности $T$. Чтобы снять эту проблему, примем следующее предположение, которое, как отмечалось в [5], означает примитивность матрицы, соответствующей подграфу $\Gamma$, составленному из дуг оптимальных контуров.

Скажем, что выполнено условие экстремальной примитивности, если для каждой из контурных компонент $\Gamma_{k}$ наибольший общий делитель количества дуг в контурах, образующих $\Gamma_{k}$, равен 1.

Условие выполняется, если контурная компонента $\Gamma_{k}$ содержит хотя бы одну петлю или хотя бы одну пару контуров с взаимно простыми количествами дуг. Это условие гарантирует для любой пары вершин $j, \tilde{j} \in \Gamma_{k}$ при любом достаточно большом $T$ наличие $T$-шагового пути из $j$ в $\tilde{j}$, лежащего в $\Gamma_{k}$.

Лемма 4. Для $i, j \in M, 0<\beta<1$

$$
\lim _{T \rightarrow \infty} V_{\beta}(i, j, T)=V_{\beta}(i)
$$

Доказателъство. Ясно, что

$$
V_{\beta}(i)=\max _{j \in M}\left\{V_{\beta}(i, j, T)+\beta^{T} V_{\beta}(j)\right\}, \quad T \geqslant 1, \quad i \in M
$$

Следовательно,

$$
V_{\beta}(i) \geqslant V_{\beta}(i, j, T)+\beta^{T} V_{\beta}(j), \quad T \geqslant 1, \quad i, j \in M
$$

С другой стороны, пусть $\Gamma_{k}-$ контурная компонента, для которой $i \in M^{-}\left(\Gamma_{k}\right)$, $\tilde{j}$ - некоторая вершина, принадлежащая $\Gamma_{k}, \pi$ - некоторый путь из $\tilde{\jmath}$ в $j$, имеющий полезность $\bar{s}(\tilde{\jmath}, j)$ и состоящий из $|\bar{s}(\tilde{\jmath}, j)|$ дуг. При предположении экстремальной примитивности для любого достаточно большого $T$ существует $T$-шаговая траектория с началом $i$ и концом $j$, совпадающая в течении первых $T-|\bar{s}(\tilde{j}, j)|$ периодов с бесконечной оптимальной траекторией с началом $i$, а затем проходящая по пути $\pi$. Полезность этой $T$-шаговой траектории равна

$$
s(i, j, T)=V_{\beta}(i)-\beta^{T-|\bar{s}(\tilde{\jmath}, j)|}\left[V_{\beta}(\tilde{\jmath})-\bar{s}(\tilde{\jmath}, j)\right] .
$$

Поскольку $V_{\beta}(i, j, T)$ - максимальная полезность $T$-шаговой траектории, из (12) и (13) следует, что

$$
V_{\beta}(i)-\beta^{T-|\bar{s}(\tilde{j}, j)|}\left(V_{\beta}(\tilde{\jmath})-\bar{s}(\tilde{\jmath}, j)\right) \leqslant V_{\beta}(i, j, T) \leqslant V_{\beta}(i)-\beta^{T} V_{\beta}(j) .
$$

Отсюда при $T \rightarrow \infty$ по теореме о сжатой переменной вытекает доказываемое утверждение. Лемма доказана.

Следствие 3. T-шаговая оптимальная траектория $\tau_{T}$ (являющаяся решением задачи $\left.S_{\beta}\left(j_{0}, j_{T}, T\right)\right)$ при достаточно большом $T$ является пошагово оптималъной на начальном участке. 
Доказательство. Пусть

$$
\tau_{T}=\left\{j_{0}, j_{1}^{T}, \ldots, j_{T-1}^{T}, j_{T}\right\}
$$

тогда

$$
j_{1}^{T} \in \operatorname{Arg} \max _{j}\left\{u\left(j_{0}, j\right)+\beta V_{\beta}\left(j, j_{T}, T-1\right)\right\} .
$$

Следовательно, в силу леммы 4 при достаточно большом $T$

$$
j_{1}^{T} \in \operatorname{Arg} \max _{j}\left\{u\left(j_{0}, j\right)+\beta V_{\beta}(j)\right\} .
$$

Утверждение доказано.

Следствие 4. Для любого натурального числа $T_{0}$ существует натуральное число $\tilde{T}$ такое, что всякая $T$-шаговая оптимальная траектория $\tau_{T}$, где $T>\tilde{T}$, на начальном участке (при $t=0,1, \ldots, T_{0}$ ) совпадает с некоторой бесконечной оптимальной траекторией с тем же начальным состоянием.

В дальнейшем, помимо экстремальной примитивности, будем всюду предполагать, что контурная компонента $\Gamma_{k}$, для которой $j_{0} \in M^{-}\left(\Gamma_{k}\right)$, единственна.

Если $T$ достаточно велико, то, как показывает следствие $4, T$-шаговая оптимальная траектория $\tau_{T}$ состоит из трех участков :

(1) переход по пути, не принадлежащему контурной компоненте $\Gamma_{k}$, из $j_{0}$ в некоторую вершину $i \in \Gamma_{k}$ (в силу следствий 3,4 этот участок совпадает с соответствующим участком бесконечной оптимальной траектории и потому представляет собой ациклический пошагово оптимальный путь ; этот участок отсутствует, если $j_{0} \in \Gamma_{k}$ );

(2) конечная траектория, соединяющая вершину $i \in \Gamma_{k}$ с некоторой вершиной $j \in \Gamma_{k}$ и целиком лежащая в контурной компоненте $\Gamma_{k}$;

(3) переход по пути, не принадлежащему контурной компоненте $\Gamma_{k}$, из $j \in \Gamma_{k}$ в $j_{T}$ (этот участок отсутствует, если $j_{T} \in \Gamma_{k}$ ).

Можно убедиться в невозможности выхода с последующим возвратом в $\Gamma_{k}$ и в отсутствии контуров на участке 3.

Таким образом, достаточно длинная $T$-шаговая оптимальная траектория представляет собой урезанную бесконечную оптимальную траекторию с началом $j_{0}$, дополненную переходом $\tau$ из некоторой вершины $j \in \Gamma_{k}$ в $j_{T}$. Пусть $s\left(j, j_{T}\right)-$ полезность пути $\tau,\left|s\left(j, j_{T}\right)\right|$ - число дуг. Значение задачи $S_{\beta}\left(j_{0}, j_{T}, T\right)$ равно

$$
V_{\beta}\left(j_{0}, j_{T}, T\right)=\max _{j \in \Gamma_{k} ; \tau}\left\{V_{\beta}\left(j_{0}\right)-\beta^{T-\left|s\left(j, j_{T}\right)\right|} V_{\beta}(j)-\beta^{T-\left|s\left(j, j_{T}\right)\right|} s\left(j, j_{T}\right)\right\} .
$$

С учетом постоянных, путь оптимального перехода из $\Gamma_{k}$ в $j_{T}$ является решением задачи

$$
\max _{j \in \Gamma_{k} ; \tau}\left\{-\beta^{-\left|s\left(j, j_{T}\right)\right|}\left(V_{\beta}(j)-s\left(j, j_{T}\right)\right)\right\} .
$$

Таким образом, при достаточно большом $T$ финальный участок траекториирешения задачи $S_{\beta}\left(j_{0}, j_{T}, T\right)$ не зависит ни от $j_{0}$ ни от $T$. 
Покажем, что любая вершина $j$ контурной компоненты $\Gamma_{k}$ может быть решением задачи (14), т. е. все вершины контурной компоненты $\Gamma_{k}$ эквивалентны для решения задач $S_{\beta}\left(j_{0}, j_{T}, T\right), j_{0} \in M^{-}\left(\Gamma_{k}\right)$, при достаточно больших $T$. Определим функцию

$$
\lambda(i)=\max _{\tau}\left\{-\beta^{-\left|s\left(i, j_{T}\right)\right|}\left(V_{\beta}(i)-s\left(i, j_{T}\right)\right)\right\}
$$

где максимум определяется по всевозможным путям $\tau$ из $i$ в $j_{T}$.

Предложение 3. Если $i_{1} \in \Gamma_{k}, i_{2} \in \Gamma_{k}$, mo $\lambda\left(i_{1}\right)=\lambda\left(i_{2}\right)$.

Доказательство. Пусть $\nu\left(i, j_{T}\right),\left|\nu\left(i, j_{T}\right)\right|$ - соответственно, полезность и число дуг для пути $\tau$, на котором достигается максимум в (15). Для произвольного пути из $i_{1}$ в $i_{2}$, лежащего в $\Gamma_{k}$, имеющего полезность $s\left(i_{1}, i_{2}\right)$ и число дуг $\left|s\left(i_{1}, i_{2}\right)\right|$, выполняется равенство

$$
V_{\beta}\left(i_{1}\right)=s\left(i_{1}, i_{2}\right)+\beta^{\left|s\left(i_{1}, i_{2}\right)\right|} V_{\beta}\left(i_{2}\right)
$$

С другой стороны, сравнивая пути с полезностями $\nu\left(i_{1}, j_{T}\right)$ и $s\left(i_{1}, i_{2}\right)+\beta^{\left|s\left(i_{1}, i_{2}\right)\right|}+$ $\left|\nu\left(i_{2}, j_{T}\right)\right|$, приходим к неравенству

$$
\lambda\left(i_{1}\right) \geqslant-\beta^{-\left|s\left(i_{1}, i_{2}\right)\right|+\left|\nu\left(i_{2}, j_{T}\right)\right|}\left(V_{\beta}\left(i_{1}\right)-s\left(i_{1}, i_{2}\right)-\beta^{\left|s\left(i_{1}, i_{2}\right)\right|} \nu\left(i_{2}, j_{T}\right)\right) .
$$

Отсюда, после подстановки $(16)$, следует, что $\lambda\left(i_{1}\right) \geqslant \lambda\left(i_{2}\right)$. Поскольку порядок $i_{1}, i_{2}$ был произволен, верно и обратное неравенство.

Предложение доказано.

Отсюда следует, что при достаточно больших $T$ существуют траектории-решения задачи $S_{\beta}\left(j_{0}, j_{T}, T\right)$, проходящие через любые вершины контурной компоненты $\Gamma_{k}$.

Общее для всех вершин $i$ контурной компоненты $\Gamma_{k}$ значение $\lambda(i)$ при фиксированном $j_{T}$ обозначим $W_{\beta}\left(j_{T}\right)$. Функция $W_{\beta}(j), j \in M$, выражает максимальную прибыль, получаемую при сходе с контурной компоненты $\Gamma_{k}$ в вершину $j$ :

$$
W_{\beta}(j)=\beta^{-|\nu(i, j)|}\left(-V_{\beta}(i)+\nu(i, j)\right) .
$$

Заметим, что множитель $\beta^{-|\nu(i, j)|}$ приводит полезность к моменту времени, следующему за моментом прихода траектории в вершину $j$.

Пусть $j_{1}, j_{2}$ - последовательные вершины некоторой $T$-шаговой оптимальной траектории после схода с контурной компоненты $\Gamma_{k}$. Тогда

$$
W_{\beta}\left(j_{2}\right)=\beta^{-1}\left(W_{\beta}\left(j_{1}\right)+u\left(j_{1}, j_{2}\right)\right) .
$$

Пусть другой путь с полезностью $\eta\left(i, \bar{j}_{1}\right)$ составляет часть некоторой $\bar{T}$-шаговой оптимальной траектории после схода с $\Gamma_{k}$. Конечная траектория, составленная из указанного пути и дуги $\left(\bar{j}_{1}, j_{2}\right)$, не обязательно оптимальна, поэтому

$$
\beta^{-\left|\eta\left(i, \bar{j}_{1}\right)\right|-1}\left(-V_{\beta}(i)+\eta\left(i, \bar{j}_{1}\right)+\beta^{\left|\eta\left(i, \bar{j}_{1}\right)\right|} u\left(j_{1}, j_{2}\right)\right) \leqslant W_{\beta}\left(i_{2}\right),
$$

T. e.

$$
W_{\beta}\left(i_{2}\right) \geqslant \beta^{-1}\left(W_{\beta}\left(\bar{j}_{1}\right)+u\left(\bar{j}_{1}, j_{2}\right)\right) \text {. }
$$


Таким образом, функция $W_{\beta}(j)$ удовлетворяет следующему уравнению Беллмана:

$$
W_{\beta}(j)=\beta^{-1} \max _{i \in M}\left\{u(i, j)+W_{\beta}(i)\right\}
$$

На контурном графе Г выполняются равенства

$$
W_{\beta}(i)=-V_{\beta}(i), \quad i \in \Gamma .
$$

Теорема 3 (о представлении функции-значения). При достаточно болъшом $T$ имеет место представление (1) для любых $j_{0}, j_{T} \in M$.

Доказательство. Зафиксируем произвольную вершину $i \in \Gamma$. Тогда

$$
\begin{aligned}
V_{\beta}\left(j_{0}\right)+\beta^{T} W_{\beta}\left(j_{T}\right)= & s\left(j_{0}, i\right)+\left(\beta^{\left|s\left(j_{0}, i\right)\right|}-\beta^{T-\left|\nu\left(i, j_{T}\right)\right|}\right) V_{\beta}(i)+\beta^{T-\left|\nu\left(i, j_{T}\right)\right|} \nu\left(i, j_{T}\right) \\
= & s\left(j_{0}, i\right)+\beta^{\left|s\left(j_{0}, i\right)\right|} c_{\sigma, i}\left(1+\beta+\ldots+\beta^{T-\left|s\left(j_{0}, i\right)\right|-\left|\nu\left(i, j_{T}\right)\right|-1}\right) \\
& +\beta^{T-\left|\nu\left(i, j_{T}\right)\right|} \nu\left(i, j_{T}\right)=V_{\beta}\left(j_{0}, j_{T}, T\right) .
\end{aligned}
$$

Теорема доказана.

Как показывает теорема, последовательность $V_{\beta}\left(j_{0}, j_{T}, T\right)$ не стабилизируется при конечных значениях $T$. В терминах [6] оператор не является нильпотентным. Тем не менее, можно вычислить приближенно значения $V_{\beta}(i), W_{\beta}(i), i \in M$, и использовать их для точного построёния $T$-шаговых оптимальных траекторий при достаточно больших $T$. Это иллюстрирует следующий пример.

\section{4. Численный пример}

Пусть $\beta=0,7, n=4$, и длины дуг $u(i, j)$ определяются матрицей

$$
U=\left(\begin{array}{llll}
1 & 4 & 2 & 5 \\
1 & 3 & 5 & 2 \\
3 & 3 & 4 & 2 \\
3 & 3 & 2 & 1
\end{array}\right)
$$

Рассмотрим последовательность степеней $U^{t}, t=1,2, \ldots$, матрицы $U$, где $U_{i j}^{t}=$ $V_{\beta}(i, j, T)$. Согласно теореме 3 , при достаточно больших $t$

$$
U_{i j}^{t}=V_{\beta}(i)+\beta^{t} W_{\beta}(j) .
$$

Отсюда при $t \rightarrow \infty$ следует сходимость

$$
U_{i j}^{t} \rightarrow V_{\beta}(i)
$$

Эти свойства позволяют легко найти с любой степенью точности значения $V_{\beta}(i)$, $W_{\beta}(i), i \in M$. Так, вычисляем рекуррентно степени $U^{t}$ и находим два значения $t$, для которых с достаточной точностью выполняются (18) и (19). В нашем примере это будут, например, $t=9$ и $t=49$.

Из $U^{49}$, используя (19), находим, что

$$
V_{\beta}(1) \approx 14,1233, \quad V_{\beta}(2) \approx 14,3333, \quad V_{\beta}(3) \approx 13,3333, \quad V_{\beta}(4) \approx 13,0333
$$


Находим также, что

$$
\beta V_{\beta}(1) \approx 9,8860, \quad \beta V_{\beta}(2) \approx 10,0333, \quad \beta V_{\beta}(3) \approx 9,3333, \quad \beta V_{\beta}(4) \approx 9,1233 .
$$

Видим, что равенства в (2) достигаются на дугах $(1,4),(2,3),(3,3),(4,2)$. Это означает, что имеется единственная контурная компонента $\Gamma$, представляющая собой петлю $(3,3)$, а пошагово оптимальные (они же оптимальные) траектории выходят на $\Gamma$ по пути $\{1,4,2,3\}$.

Теперь из $U^{9}$, используя найденные $V_{\beta}(i)$, по равенствам (18), находим, что

$$
\begin{aligned}
& W_{\beta}(1)=W_{\beta}(2) \approx-14,7619, \quad W_{\beta}(3) \approx-13,3333, \quad W_{\beta}(4) \approx-13,9456 ; \\
& \beta W_{\beta}(1)=\beta W_{\beta}(2) \approx-10,3333, \quad \beta W_{\beta}(3) \approx-9,3333, \quad \beta W_{\beta}(4) \approx-9,7619 .
\end{aligned}
$$

Видим, что равенства в $(17)$ достигаются на дугах $(3,1),(3,2),(3,3),(1,4)$. Это означает, что сход достаточно длинных $T$-шаговых траекторий с контурной компоненты $\Gamma$ происходит по путям $\{3,1,4\}$ и $\{3,2\}$.

Получено полное описание любоћ $T$-шаговой оптимальной траектории при достаточно большом $T$. Например, траектория-решение задачи $S_{\beta}(1,4, T)$ имеет следующую структуру : выход на контурную компоненту по пути $\{1,4,2,3\}$; обход оптимального контура (петли $(3,3)) T-5$ раз; переход в вершину 4 по пути $\{3,1,4\}$.

\section{5. Случай $\beta>1$}

$\mathrm{K}$ исследованию задачи $S_{\beta}\left(j_{0}, j_{T}, T\right)$ для случая $\beta>1$ можно применить результаты, полученные выше для случая $\beta<1$. Для этого введем понятие обратной траектории.

Последовательность

$$
\tau=\left\{\ldots, i_{k-t}, \ldots, i_{k-2}, i_{k-1}, i_{k}\right\}
$$

называется (бесконечнои) обратной траекторией с началом $i_{k}$, если

$$
u\left(i_{h-1}, i_{h}\right)>-\infty, \quad h=k, k-1, \ldots
$$

Конечный участок

$$
\left\{i_{k-T}, i_{k-T+1}, \ldots, i_{k-1}, i_{k}\right\}
$$

называется $T$-шаговой обратной траекторией с началом $i_{k}$ и концом $i_{k-T}$.

Положим

$$
\delta=\beta^{-1} .
$$

Очевидно, что $0<\delta<1$ при $\beta>1$. Задача $S_{\beta}\left(j_{0}, j_{T}, T\right)$ эквивалентна задаче $\tilde{S}_{\delta}\left(j_{0}, j_{T}, T\right)$ нахождения $T$-шаговой обратной траектории с началом $j_{T}$ и концом $j_{0}$, максимизирующей функцию

$$
\sum_{k=0}^{T-1} \delta^{k} u\left(i_{T-k-1}, i_{T-k}\right) .
$$

Пусть, как и раньше, $V_{\beta}\left(j_{0}, j_{T}, T\right)$ - значение задачи $S_{\beta}\left(j_{0}, j_{T}, T\right)$. Через $\tilde{V}_{\delta}\left(j_{0}, j_{T}, T\right)$ обозначим значение задачи $\tilde{S}_{\delta}\left(j_{0}, j_{T}, T\right)$. Нетрудно видеть, что

$$
V_{\beta}\left(j_{0}, j_{T}, T\right)=\beta^{T-1} \tilde{V}_{\delta}\left(j_{0}, j_{T}, T\right) .
$$


Теорема 4. При $\beta>1$ существуют тахие наборы иисел $\mathscr{V}_{\beta}(i), \mathscr{W}_{\beta}(i), i \in M$, ито

$$
\begin{aligned}
& \mathscr{V}_{\beta}(i)=\max _{j \in M}\left\{u(i, j)+\beta \mathscr{V}_{\beta}(j)\right\}, \\
& \mathscr{W}_{\beta}(j)=\beta^{-1} \max _{i \in M}\left\{u(i, j)+\mathscr{W}_{\beta}(i)\right\},
\end{aligned}
$$

и при достаточно болъиих $T$

$$
V_{\beta}\left(j_{0}, j_{T}, T\right)=\mathscr{V}_{\beta}\left(j_{0}\right)+\beta^{T} \mathscr{W}_{\beta}\left(j_{T}\right), \quad j_{0}, j_{T} \in M
$$

Доказательство состоит в применении теоремы 3 к функции $\tilde{V}_{\delta}\left(j_{0}, j_{T}, T\right)$ и последующем переходе к $V_{\beta}\left(j_{0}, j_{T}, T\right)$.

Все, сказанное выше при $\beta<1$ по поводу структуры оптимальных $T$-шаговых траекторий, переносится при $\beta>1$ на обратные оптимальные $T$-шаговые траектории.

\section{Список литературы}

1. Романовский И. В. Оптимизация стационарного управления дискретным детерминированным процессом. Кибернетика (1967) №2, 66-78.

2. Романовский И. В. Алгоритмы решения эхстремалъных задач. Наука, Москва, 1977.

3. Дудников П. И., Самборский С. Н. Эндоморфизмъ полумодуля над полуколвцом $c$ идемпотентной операчией. Препринт. ИМ АН УССР, Киев, 1987.

4. Матвеенко В. Д. Применение модели Неймана для исследования схемы динамического программирования. Автоматиха и телемеханиха (1988) №11, 62-70.

5. Матвеенко В. Д. Оптимальные траектории схемы динамического программирования и эхстремальные степени неотрицательных матриц. Дискретная математика (1990) 2, №1, 59-71.

6. Маслов В. П., Колокольцев В. Н. Идемпотентяый анализ и его применение в оптималъном управлении. Физматлит, Москва, 1994.

7. Матвеенко В. Д. Плановые и рыночные механизмы управления в моделях экономическои динамихи. Препринт. ИСЭП АН СССР, Ленинград, 1989.

8. Беленький В. З. Экономическая динамика: обобщающая бюджетная факторизация гейовской технологии. Эхономиха и математические методъ (1990) 26, №1, 165177.

9. Ашманов С. А. Введение в математическую экономику. Наука, Москва, 1984.

10. Takayama A. Mathematical Economics. Cambridge University Press, Cambridge, 1985.

11. McKenzie L. W. Optimal economic growth and Turnpike theorems. In: Handbook of Mathematical Economics. Vol. 3, North Holland, Amsterdam, 1986.

12. Boldrin M., Montrucchio L. Acyclicity and dynamic stability: generalizations and applications. J. Economic Theory (1995) 65, №2, 303-326.

13. Dutta P. K. What do discounted optima converge to? A theory of discount rate asymptotics in economic models. J. Economic Theory (1991) 55, №1, 64-94.

14. Fudenberg D., Maskin E. The folk theorem in repeated games with discounting and with incomplete information. Econometrica (1986) 54, 533-554.

15. Романовский И. В. Асимптотическое поведение дискретного детерминированного процесса с непрерывным множеством состояний. Оптимальное планирование (1967) №8, 171-193. 
16. Stokey N. L., Lucas R. E. Recursive Methods in Economic Dynamics. Harvard Univ. Press, Cambridge, 1989.

17. Deneckere R., Pelican S. Competitive chaos. J. Economic Theory (1986) 40, №1, 13-25.

18. Boldrin M., Montrucchio L. On the indeterminacy of capital accumulation paths. J. Economic Theory (1986) 40, №1, 26-39.

19. Boldrin M., Montrucchio L. Acyclicity and stability for intertemporal optimization models. International Economic Review (1988) 29, №1, 137-146.

Статья поступила 15.11.95. 